\title{
Antiepidermal Growth Factor Receptor Monoclonal Antibodies: Applications in Colorectal Cancer
}

\author{
Efat Azizi, ${ }^{1,2}$ Adam Kittai, ${ }^{1,2}$ and Peter Kozuch ${ }^{1,2}$ \\ ${ }^{1}$ Beth Israel Medical Center, Phillips Ambulatory Care Center, Continuum Cancer Centers of New York, 10 Union Square East, \\ Suite 4C, New York, NY, USA \\ ${ }^{2}$ Section of Hematology/Oncology, Department of Medicine, Albert Einstein College of Medicine, Bronx, NY, USA
}

Correspondence should be addressed to Efat Azizi, eazizi@chpnet.org

Received 16 April 2012; Accepted 12 June 2012

Academic Editor: Basil El-Rayes

Copyright (C) 2012 Efat Azizi et al. This is an open access article distributed under the Creative Commons Attribution License, which permits unrestricted use, distribution, and reproduction in any medium, provided the original work is properly cited.

\begin{abstract}
Patients with metastatic colorectal cancer have a poor prognosis and present a challenge to clinicians. The role of the antiepidermal growth factor receptor (EGFR) pathway in tumorogenesis and tumor progression has been well defined. This paper will review the use of anti-EGFR monoclonal antibodies in the treatment of operable, as well as metastatic colorectal cancer both in the setting of KRAS mutation unselected patients and later in KRAS wild-type patients. Active investigations designed to further identify predictive biomarkers that may be potentially druggable are reviewed as well.
\end{abstract}

\section{Introduction}

Colorectal cancer is the third most commonly diagnosed cancer and the third leading cause of cancer death in both men and women in the US [1]. It is estimated that over 140,000 new cases of colorectal cancer (CRC) were diagnosed in 2011 and approximately 50,000 died of this disease [2]. Until a little more than a decade ago the only drug approved for this disease was fluorouracil (5FU), and the median survival with stage IV disease was 12 months. With the development of drugs such as irinotecan and oxaliplatin, the median survival associated with this disease has increased to over 20 months. The ongoing development of antiepidermal growth factor receptor (EGFR) agents and the identification of predictive markers to identify enriched populations who will benefit from anti-EGFR therapy represent active areas of clinical and translational research. This paper will acquaint readers with the pathophysiology that guided the development of anti-EGFR therapies for colorectal cancer and will synthesize the huge amount of clinical data that supports limiting the use of cetuximab and panitumumab alone or in combination with irinotecan as second- or third-line therapy for metastatic colorectal cancer without mutations of the KRAS gene.

\section{EGFR}

The EGFR is a cell surface 170,000 dalton tyrosine kinase transmembrane receptor and a member of the human epidermal growth factor receptor (HER)-ErbB family of receptor tyrosine kinases [3]. Dysregulation of the EGFR pathway occurs in a variety of ways including genetic mutation, gene amplification, protein overexpression, structural rearrangement, and autocrine ligand production [4].

The ErbB family is composed of 4 transmembrane receptors that interact with each other: EGFR/ErbB1/Her1, ErbB2/Her2/neu, ErbB3/Her3, and ErbB4/Her4 [3-5]. This interaction can result in either homodimerization or heterodimerization. Following dimerization, the intracellular tyrosine kinase portion is phosphorylated leading to downstream activation of complex interacting signaling pathways which include the Ras/Raf/MEK/ERK and the Ras/PI13 K/PTEN//AKT/mTOR pathways [5]. These pathways have been shown to regulate cellular replication, invasion, cellular repair, protection from insult, and induction of apoptosis. As diagrammed in Figure 1, signaling is thought to operate via both vertical and horizontal pathways. As intracellular signaling is found to be a vastly complex network, there is increasing rationale to target more than one 


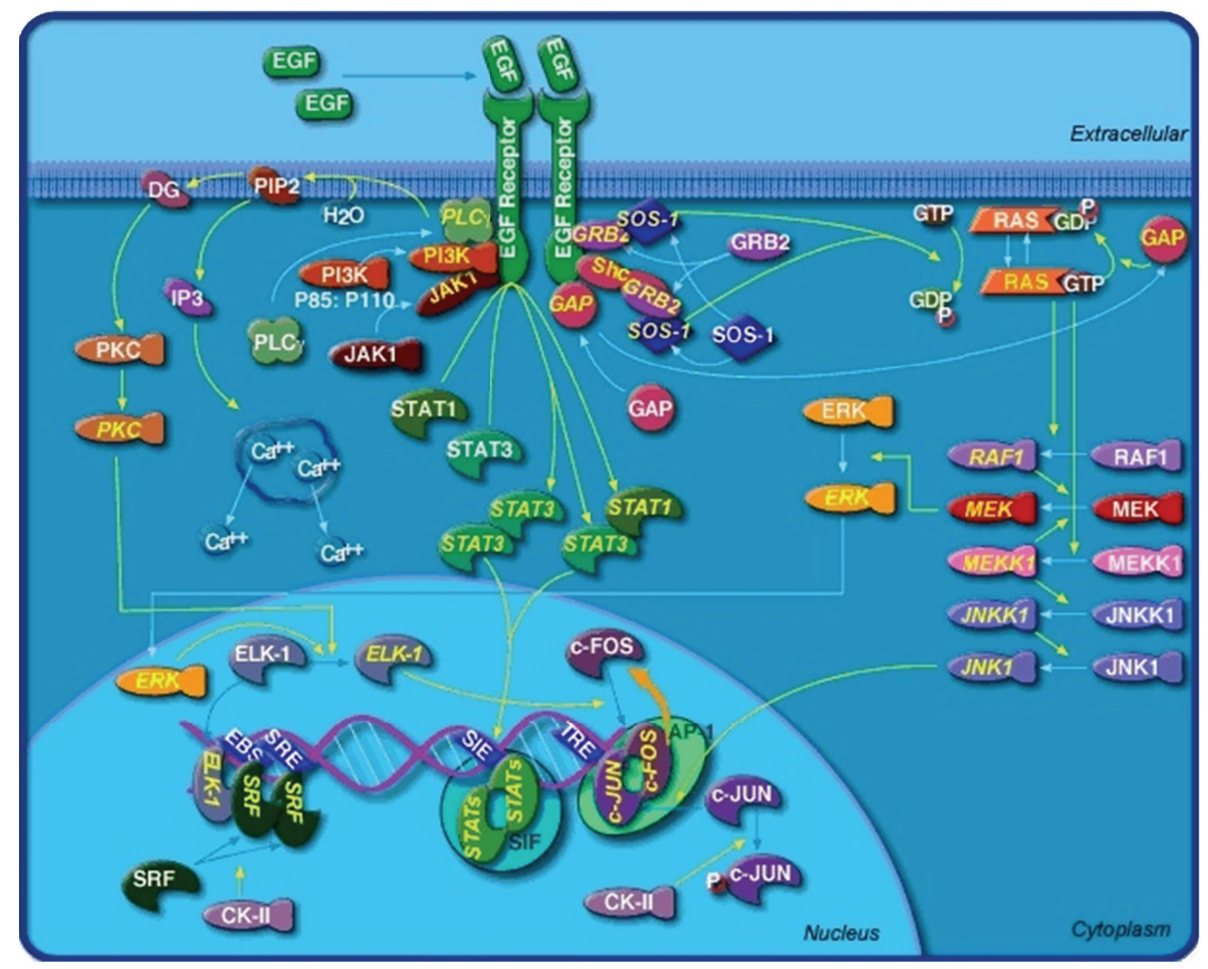

FIGURE 1: EGFR signaling pathway (reprinted with permission from BioCarta Pathways. All rights reserved).

signaling pathway or multiple targets within a single pathway in order to effectively regulate cancer. The design of an anticancer therapy employing an inhibitor of EGFR function was hypothesis-driven, based on knowledge available in the early 1980s [6]. EGFR and the Src oncogene product were shown to have the novel enzymatic activity of a tyrosine kinase [6]. Subsequent studies established that EGFR was a cellular oncogene and demonstrated that high levels of EGFR correlated with poorer prognosis in solid tumors [6]. Preclinical studies hypothesized that blockade of the EGFR binding sites with an "antireceptor" monoclonal antibody $(\mathrm{mAb})$ would lead to the inhibition of cell growth, thereby making it an effective anticancer therapy [6].

\section{EGFR Antagonists}

There are two classes of EGFR antagonists currently in clinical use: anti-EGFR monoclonal antibodies and EGFR tyrosine kinase inhibitors (TKIs) [5] (Figure 2). Initial clinical trials of these agents did not assess subjects' tumors for the absence of KRAS mutations which have since been found to confer resistance to anti-EGFR mAbs. Restricting eligibility for clinical trial participation to only patients with wildtype KRAS (wtKRAS) CRC as opposed to mutated KRAS (mutKRAS) CRC has been a crucial step in optimizing the use of EGFR targeting mAbs. Cetuximab and panitumumab are the two anti-EGFR mAbs that have demonstrated clinical benefit and have gained FDA approval for the palliative treatment of chemotherapy resistant wtKRAS metastatic colorectal cancer (mCRC). Both mAbs bind to the extracellular domain of the cell receptor and inhibit dimerization, tyrosine kinase activation, and subsequent cell signal transduction [5].

Cetuximab is a human-murine chimeric monoclonal antibody that binds to EGFR with high specificity and with a higher affinity than the natural ligands epidermal growth factor or TGF-0 $[3,12]$. Thus, the mechanism of action is thought to be inhibition of ligand induced phosphorylation of EGFR [5]. Inhibition of natural ligand binding to EGFR results in several different downstream effects, all of which may contribute to the antitumor activity seen with cetuximab [4]. Cell growth and cell proliferation are turned off, apoptosis is induced, and EGFR is downregulated by internalization and degradation. Cetuximab also has been shown to decrease production of matrix metalloproteinases, enzymes which have been linked to metastatic potential [4]. Panitumumab was the first fully humanized IgG2 $\mathrm{mAb}$ directed against EGFR. The mechanism of action of panitumumab is similar to that of cetuximab. Panitumumab binds to EGFR, thereby preventing receptor dimerization and activation of downstream molecular signaling [9].

\section{Cetuximab Monotherapy in Chemotherapy Refractory CRC Not Selected by KRAS Status (Table 1)}

The clinical development of cetuximab predated panitumumab. Similar to many new drugs, cetuximab and panitumumab were initially evaluated as single agents in patients with mCRC resistant to all available cytotoxic chemotherapy agents. The first trial demonstrating that treatment with 


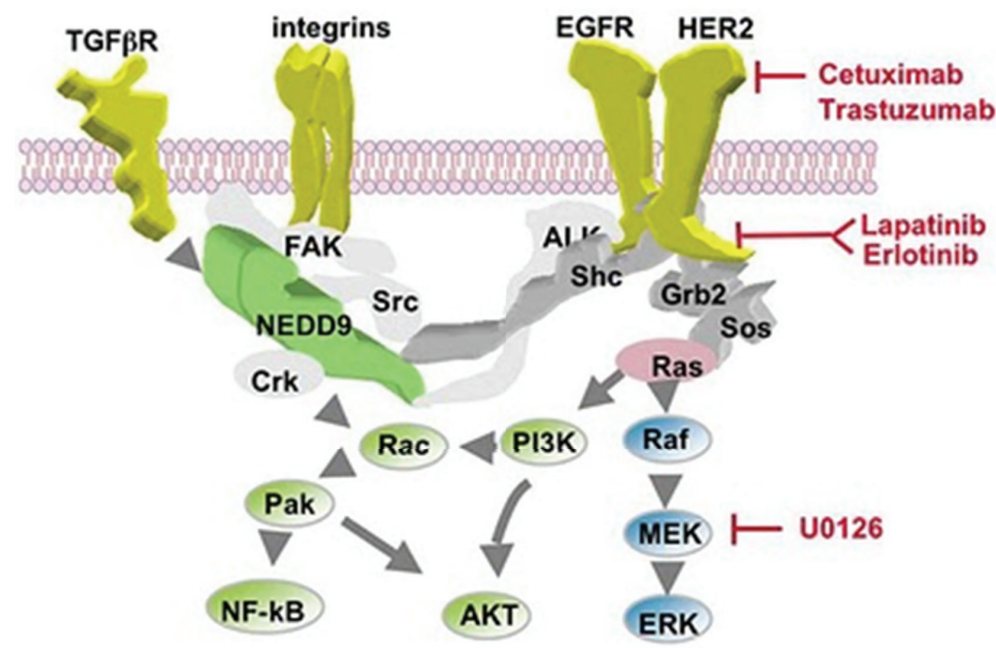

Figure 2: Reprinted with permission from Erica A. Golemis, Ph. D. All rights reserved.

TABle 1: Trials using Anti-EGFR mABs for chemotherapy-resistant mCRC not selected by KRAS status.

\begin{tabular}{|c|c|c|c|c|c|c|c|}
\hline \multirow{2}{*}{ Trial (author) } & \multirow{2}{*}{ Phase } & \multirow{2}{*}{ Protocol } & \multirow{2}{*}{$\begin{array}{l}\text { Number } \\
\text { enrolled }\end{array}$} & \multicolumn{2}{|c|}{ Results } & \multirow[b]{2}{*}{ HR (95\% CI) } & \multirow{2}{*}{$P$ value } \\
\hline & & & & PFS & OS & & \\
\hline - (Saltz et al.) [7] & II & Cetuximab & 57 & \multicolumn{2}{|c|}{ PR 9\% } & & \\
\hline CO.17 (Jonker et al.) [8] & III & $\begin{array}{l}\text { Cetuximab } \\
\text { BSC }\end{array}$ & $\begin{array}{l}287 \\
285\end{array}$ & \multicolumn{2}{|r|}{$\begin{array}{l}6.1 \text { mos. } \\
4.6 \text { mos. }\end{array}$} & OS: $0.77(0.64-0.92)$ & $<0.005$ \\
\hline — (Peeters et al.) [9] & III & $\begin{array}{c}\text { Panitumumab }+ \text { BSC } \\
\text { BSC }\end{array}$ & $\begin{array}{l}231 \\
232\end{array}$ & \multicolumn{2}{|l|}{$\begin{array}{l}8 \text { wks. } \\
7.3 \text { wks. }\end{array}$} & PFS: $0.54(0.44-0.66)$ & $<0.0001$ \\
\hline $\begin{array}{l}\text { BOND (Cunningham } \\
\text { et al.) [10] }\end{array}$ & II & $\begin{array}{c}\text { Cetuximab }+ \text { irinotecan } \\
\text { cetuximab }\end{array}$ & $\begin{array}{l}218 \\
211\end{array}$ & \multicolumn{2}{|c|}{$\begin{array}{l}\text { PR } 22.9 \% \\
\text { PR } 10.8 \%\end{array}$} & PR: N/A & $<0.001$ \\
\hline \multirow[t]{2}{*}{$\begin{array}{l}\text { EPIC (Sobrero et al.) } \\
{[11]}\end{array}$} & \multirow[t]{2}{*}{ III } & Cetuximab + Irinotecan & 648 & $\begin{array}{r}4.0 \text { mos. } \\
\text { RR } \\
2.6 \text { mos. }\end{array}$ & $\begin{array}{l}10.7 \text { mos. } \\
4 \% \\
10.0 \text { mos. }\end{array}$ & \multirow[t]{2}{*}{$\begin{array}{l}\text { PFS: } 0.69(0.61-0.77) \\
\text { OS: } 0.97(0.84-1.11) \\
\text { RR: N/A }\end{array}$} & \multirow[t]{2}{*}{$\begin{array}{l}<0.0001 \\
0.71 \\
<0.0001\end{array}$} \\
\hline & & & & & & & \\
\hline
\end{tabular}

BSC: best supportive care, PR: partial response, OS: overall survival, PFS: progression-free survival, RR: response rate, and N/A: not applicable.

cetuximab alone was active in patients refractory to cytotoxic chemotherapy was reported by Saltz et al. in 2004. Patients were required to have disease resistance to irinotecan or an irinotecan containing regimen. Fifty-seven patients were treated and response rate was the only reported outcome in this phase II trial. Nine percent of subjects attained a partial response (PR) [7].

In 2007 Jonker et al. published results of a phase III trial looking at cetuximab or best supportive care (BSC) in patients refractory to fluoropyrimidine, irinotecan, and oxaliplatin. This trial is particularly notable due to the fact that it demonstrated an OS benefit in association with cetuximab. Patients were randomized to receive weekly infusions of cetuximab or BSC alone. In comparison with BSC, cetuximab treatment was associated with an improvement in OS (6.1 mos. versus 4.6 mos.: hazard ratio (HR) for death,
$0.77 ; 95 \%$ confidence interval (CI), 0.64 to $0.92 ; P=0.005$ ) [8]. It is important to note that these studies were performed prior to the identification of KRAS mutations as predictors of anti-EGFR mAb resistance.

\section{Panitumumab Monotherapy in Chemotherapy Refractory CRC Not Selected by KRAS Status (Table 1)}

An open-label phase III trial of panitumumab plus BSC versus BSC alone in patients with mCRC refractory to chemotherapy demonstrated a mean PFS of 8 weeks for panitumumab and 7.3 weeks for BSC ( $\mathrm{HR} 0.54 ; 95 \% \mathrm{CI}$, 0.44 to $0.66, P<0.0001)$. Twenty-two (10\%) patients in the panitumumab group attained a $\mathrm{PR}$ with a median response 
TABLE 2: Trials using Anti-EGFR mABs as monotherapy for chemoresistant mCRC selected by KRAS status.

\begin{tabular}{|c|c|c|c|c|c|c|c|c|}
\hline \multirow{2}{*}{ Trial (author) } & \multirow{2}{*}{ Phase } & \multirow{2}{*}{ KRAS } & \multirow{2}{*}{ Protocol } & \multirow{2}{*}{$\begin{array}{l}\text { Number } \\
\text { enrolled }\end{array}$} & \multicolumn{2}{|c|}{ Results } & \multirow{2}{*}{$\operatorname{HR}(95 \% \mathrm{CI})$} & \multirow{2}{*}{$P$ value } \\
\hline & & & & & PFS & OS & & \\
\hline \multirow{4}{*}{ — (Amado et al.) [14] } & \multirow{4}{*}{ III } & & Panitumumab & 124 & 12.3 wks. & 8.1 mos. & PFS: $0.45(0.34-0.59)$ & $<0.001$ \\
\hline & & WT & BSC & 119 & 7.3 wks. & 7.6 mos. & OS: 0.99 (0.75-1.29) & NR \\
\hline & & & Panitumumab & 84 & 7.4 wks. & 4.9 mos. & PFS: $0.99(0.73-1.36)$ & NR \\
\hline & & MUT & BSC & 100 & 7.3 wks. & 4.4 mos. & OS: $1.02(0.75-1.39)$ & NR \\
\hline
\end{tabular}

BSC: best supportive care, WT: wild type, MUT: mutated, and NR: not reported.

duration of 17 weeks. Importantly, crossover was allowed as part of the study design and 176 BSC patients (76\%) subsequently received panitumumab which likely prevented a survival benefit to emerge [9].

\section{Cetuximab Plus Chemotherapy for Chemoresistant mCRC Not Selected by KRAS Status (Table 1)}

Chronologically, the next clinical trial efforts designed to optimize the use of anti-EGFR mAbs assessed the feasibility and benefit of concurrent chemotherapy plus mAbs. The BOND trial compared the combination of cetuximab plus irinotecan to cetuximab alone in patients with irinotecan refractory CRC. Response activity favored the combination arm, $22.9 \% \mathrm{PR}$ versus a $10.8 \% \mathrm{PR}$ in the cetuximab alone $\operatorname{arm}(P \leq 0.001)$. Progression free survival also favored the combination arm: 4.1 versus 1.5 months [10]. While monotherapy did demonstrate efficacy in this trial, the better RR and PFS support the combination for this patient population [10]. This finding also suggests that cetuximab overcomes irinotecan resistance. This may occur by weakening efflux of irinotecan, impairing DNA repair activity and restoring drug-induced apoptosis.

In an open-label phase III trial (EPIC) in patients with mCRC resistant to first-line fluorinated pyrimidines and oxaliplatin, Sobrero et al. found that concurrent cetuximab plus irinotecan compared with irinotecan monotherapy was associated with both improved progression-free survival (PFS) (4.0 versus 2.6 mos; HR, 0.692; 95\% CI 0.617-0.776; $P<0.0001)$ and response rate (RR) (16.4\% versus $4.2 \%$; $P<0.001$ ). Overall survival (OS) however, 10.7 months versus 10 months was comparable between the two groups. The authors suggested that the lack of survival difference may have been due to the fact that $46.9 \%$ of patients in the irinotecan group ultimately were treated with cetuximab upon disease progression [11].

\section{Development of KRAS as a Predictive Marker}

RAS proteins are members of the superfamily of small GTPbinding proteins otherwise known as RAS-like GTPases. These proteins are involved in signal transduction across membranes, particularly those induced by growth factors [13]. KRAS, the human homolog of the Kirsten rat sarcoma2 virus oncogene encodes a small GTP binding protein that acts as a self-inactivating signal transducer by cycling from GDP- to GTP-bound states in response to stimulation of cell surface receptors including EGFR [14]. KRAS can harbor oncogenic mutations that yield a constitutively active protein. Such mutations are found in approximately $30 \%$ to $50 \%$ of CRC tumors and are common in other tumor types [14]. KRAS mutations are currently the most recognized molecular predictive markers in CRC and predict the efficacy of anti-EGFR antibodies [15]. In 2006, Lièvre et al. reported that in a screening analysis for KRAS, BRAF, and PIK3CA mutations by direct sequencing tumors from 30 cetuximab treated mCRC patients wtKRAS survival compared with mutKRAS was significantly higher (16.3 versus 6.9 months; $P=0.016)[16]$.

\section{Anti-EGFR mAB Monotherapy for Chemoresistant mCRC Selected by KRAS Status (Table 2)}

Subsequently, when KRAS mutational status as a predictor of anti-EGFR mAb benefit was retrospectively assessed among $92 \%$ of 463 subjects enrolled in a prospective trial of panitumumab monotherapy versus BSC, clinical benefit was limited to wtKRAS patients. Wild-type KRAS status was associated with significantly better PFS (12.3 weeks versus 7.3 weeks) and RR (17\% versus $0 \%)$. Also, in multivariate analysis wtKRAS patients had a longer OS ( HR, 0.67; 95\% CI, 0.55 to 0.82 ; treatment arms combined). The observed difference in OS between wtKRAS and mutKRAS was driven by survival benefit in wtKRAS patients who crossed over to panitumumab upon disease progression in the BSC arm [14].

These findings lead investigators to design trials that utilized KRAS mutational status as a tool to better identify groups of patients who would benefit from anti-EGFR therapy. Universal evaluation of KRAS mutational status to appropriately select $\mathrm{mCRC}$ patients for anti-EGFR targeted therapy also became a community oncology best practice. In 2009, the American Society for Clinical Oncology published a provisional clinical opinion in which it recommended evaluation of KRAS mutational analysis in all mCRC patients and restriction of anti-EGFR antibody therapy to wtKRAS tumors [17].

\section{Anti-EGFR mAbs Plus Chemotherapy in Chemotherapy-Resistant mCRC Selected by KRAS Status (Table 3)}

Trials evaluating chemotherapy combined with anti-EGFR $\mathrm{mAbs}$ in this patient population have had mixed results. 
TABLE 3: Trials using Anti-EGFR mABs with chemotherapy in chemoresistant mCRC selected by KRAS status.

\begin{tabular}{|c|c|c|c|c|c|c|c|c|}
\hline \multirow{2}{*}{ Trial (author) } & \multirow{2}{*}{ Phase } & \multirow{2}{*}{ KRAS } & \multirow{2}{*}{ Protocol } & \multirow{2}{*}{$\begin{array}{l}\text { Number } \\
\text { enrolled }\end{array}$} & \multicolumn{2}{|c|}{ Results } & \multirow{2}{*}{$\operatorname{HR}(95 \% \mathrm{CI})$} & \multirow{2}{*}{$P$ value } \\
\hline & & & & & PFS & OS & & \\
\hline $\begin{array}{l}\text { PICCOLO } \\
\text { (Seymour et al.) } \\
{[18]}\end{array}$ & III & $\begin{array}{l}\text { WT } \\
\text { WT }\end{array}$ & $\begin{array}{l}\text { Irinotecan + panitumumab } \\
\text { Irinotecan }\end{array}$ & $\begin{array}{l}\text { Total number of } \\
\text { patients }=324\end{array}$ & & $\begin{array}{l}10.4 \text { mos. } \\
10.5 \text { mos. }\end{array}$ & OS: 0.91 (0.73-1.14) & 0.44 \\
\hline \multirow{2}{*}{$\begin{array}{l}\text { Study } 181 \\
\text { (Sobrero et al.) } \\
{[19]}\end{array}$} & \multirow[t]{2}{*}{ III } & WT & $\begin{array}{c}\text { FOFIRI + panitumumab } \\
\text { FOLFIRI }\end{array}$ & $\begin{array}{l}303 \\
294\end{array}$ & $\begin{array}{l}6.7 \text { mos. } \\
4.9 \text { mos. }\end{array}$ & $\begin{array}{l}14.5 \text { mos. } \\
12.5 \text { mos. }\end{array}$ & $\begin{array}{l}\text { PFS: } 0.82(0.69-0.97) \\
\text { OS: } 0.92(0.78-1.10)\end{array}$ & $\begin{array}{l}0.023 \\
0.366\end{array}$ \\
\hline & & MUT & $\begin{array}{c}\text { FOFIRI + panitumumab } \\
\text { FOLFIRI }\end{array}$ & $\begin{array}{l}238 \\
248\end{array}$ & $\begin{array}{l}5.3 \text { mos. } \\
5.4 \text { mos. }\end{array}$ & $\begin{array}{l}11.8 \text { mos. } \\
11.1 \text { mos. }\end{array}$ & $\begin{array}{l}\text { PFS: } 0.95(0.78-1.14) \\
\text { OS: } 0.93(0.77-1.13)\end{array}$ & $\begin{array}{l}0.561 \\
0.482\end{array}$ \\
\hline
\end{tabular}

WT: wild type, MUT: mutated, PFS: progression free survival, and OS: overall survival.

TABLE 4: Anti-EGFR mAbs monotherapy or in combination with chemotherapy for therapy naïve mCRC selected by KRAS.

\begin{tabular}{|c|c|c|c|c|c|c|c|c|}
\hline \multirow{2}{*}{ Trial (author) } & \multirow{2}{*}{ Phase } & \multirow{2}{*}{ KRAS } & \multirow{2}{*}{ Protocol } & \multirow{2}{*}{$\begin{array}{l}\text { Number } \\
\text { enrolled }\end{array}$} & \multicolumn{2}{|c|}{ Results } & \multirow{2}{*}{$\mathrm{HR}(95 \% \mathrm{CI})$} & \multirow{2}{*}{$P$ value } \\
\hline & & & & & PFS & OS & & \\
\hline \multirow{4}{*}{$\begin{array}{l}\text { Crystal } \\
\text { (Van Cutsem et al.) } \\
{[20]}\end{array}$} & \multirow{4}{*}{ III } & \multirow[t]{2}{*}{ WT } & FOLFIRI + cetuximab & 172 & 9.9 mos. & 24.9 mos. & PFS: 0.68 (0.50-0.94) & 0.07 \\
\hline & & & FOLFIRI & 176 & 8.7 mos. & 21.0 mos. & OS: $0.84(0.64-1.11)$ & NR \\
\hline & & \multirow{2}{*}{ MUT } & FOLFIRI + cetuximab & 105 & 7.6 mos. & 17.5 mos. & PFS: 1.07 (0.71-1.61) & 0.44 \\
\hline & & & FOLFIRI & 87 & 8.1 mos. & 17.7 mos. & OS: $1.03(0.74-1.44)$ & NR \\
\hline \multirow{2}{*}{$\begin{array}{l}\text { OPUS } \\
\text { (Bokemeyer et al.) } \\
{[21]}\end{array}$} & \multirow[t]{2}{*}{ II } & WT & $\begin{array}{l}\text { FOLFOX + cetuximab } \\
\text { FOLFOX }\end{array}$ & $\begin{array}{l}61 \\
73\end{array}$ & $\begin{array}{l}7.7 \text { mos. } \\
7.2 \text { mos. }\end{array}$ & & PFS: 0.57 (0.35-0.90) & 0.0163 \\
\hline & & MUT & $\begin{array}{l}\text { FOLFOX }+ \text { cetuximab } \\
\text { FOLFOX }\end{array}$ & $\begin{array}{l}52 \\
47\end{array}$ & $\begin{array}{l}5.5 \text { mos. } \\
8.6 \text { mos. }\end{array}$ & & PFS: 1.83 (1.09-3.05) & 0.192 \\
\hline \multirow{2}{*}{$\begin{array}{l}\text { PRIME } \\
\text { (Douillard et al.) } \\
{[22]}\end{array}$} & \multirow[t]{2}{*}{ III } & WT & $\begin{array}{c}\text { FOLFOX + panitumumab } \\
\text { FOLFOX }\end{array}$ & $\begin{array}{l}325 \\
331\end{array}$ & $\begin{array}{l}9.6 \text { mos. } \\
8.0 \text { mos. }\end{array}$ & $\begin{array}{l}23.9 \text { mos. } \\
19.7 \text { mos. }\end{array}$ & $\begin{array}{l}\text { PFS: } 0.80(0.66-0.97) \\
\text { OS: } 0.83(0.67-1.01)\end{array}$ & $\begin{array}{c}0.02 \\
0.072\end{array}$ \\
\hline & & MUT & $\begin{array}{c}\text { FOLFOX + panitumumab } \\
\text { FOLFOX }\end{array}$ & $\begin{array}{l}221 \\
219\end{array}$ & $\begin{array}{l}7.3 \text { mos. } \\
8.8 \text { mos. }\end{array}$ & $\begin{array}{l}15.5 \text { mos. } \\
19.3 \text { mos. }\end{array}$ & $\begin{array}{l}\text { PFS: } 1.29(1.04-1.62) \\
\text { OS: } 1.24(0.98-1.57)\end{array}$ & $\begin{array}{l}0.02 \\
0.68\end{array}$ \\
\hline
\end{tabular}

WT: wild type, MUT: mutated, PFS: progression free survival, OS: overall survival, and NR: not reported.

The PICCOLO trial randomized patients with wtKRAS mCRC resistant to one or more prior chemotherapy regimens to treatment with irinotecan plus panitumumab or irinotecan alone. This study did not meet its primary endpoint of improved OS, but a trend was seen toward survival benefit beyond 12 months, especially in wtKRAS/wtBRAF. Patients with BRAF-mutated tumors actually had significant disbenefit with panitumumab [18]. The putative mechanism of resistance of BRAF mutations and BRAF as a clinical predictor of EGFR targeted therapy is detailed later in this paper. Sobrero et al. compared (fluorouracil, leucovorin, and irinotecan) FOLFIRI alone with FOLFIRI plus panitumumab as second-line treatment in 597 subjects. Median PFS (6.7 versus $4.9 ; P=0.023$ ) favored the panitumumab arm. Median OS, however, (14.5 versus $12.5, P=0.366$ ) was not improved with panitumumab, possibly because of postprogression EGFR targeted treatment received by 35\% of subjects on the FOLFIRI alone control arm [19].

\section{Anti-EGFR mAbs Monotherapy or in Combination with Chemotherapy for Therapy Naïve mCRC Selected by KRAS (Table 4)}

The CRYSTAL trial compared FOLFIRI alone with FOLFIRI in combination with cetuximab as treatment of therapy naïve mCRC. This study concluded that first-line treatment with cetuximab plus FOLFIRI, as compared to FOLFIRI alone, reduced the risk of PFS only in patients with wtKRAS tumors. The hazard ratio for PFS was 0.68 (95\% CI, 0.5 to 0.94 ) in patients with wtKRAS tumors and favored the investigational arm [20]. Unfortunately, the extended PFS was not translated to an improved OS. A smaller phase II trial, OPUS, in the same patient population compared FOLFOX alone to FOLFOX with cetuximab. There was a longer PFS $(7.7$ mos. versus 7.3 mos.; $P=0.0163)$ in the cetuximab arm but there was no difference in OS [21]. Again wtKRAS patients were the ones that derived benefit from the mAb treatment. In 2009, the PRIME trial confirmed that when administered as first-line therapy, panitumumab in combination with FOLFOX prolonged PFS in wtKRAS mCRC patients $(9.6$ mos. versus 8.0 mos.; $P=$ 0.02 ). There was a nonsignificant increase OS observed in the investigational arm [22]. These trials therefore do not support the use of anti-EGFR mAbs as part of first-line therapy for mCRC.

\section{Anti-EGFR mAbs Combined with anti-VEGF mAbs Selected by KRAS}

Additional clinical development of anti-EGFR mAbs included investigations of these mAbs in combination with 
TABLE 5: Trials using anti-EGFR mAbs plus bevacizumab in chemotherapy-resistant mCRC not selected by KRAS.

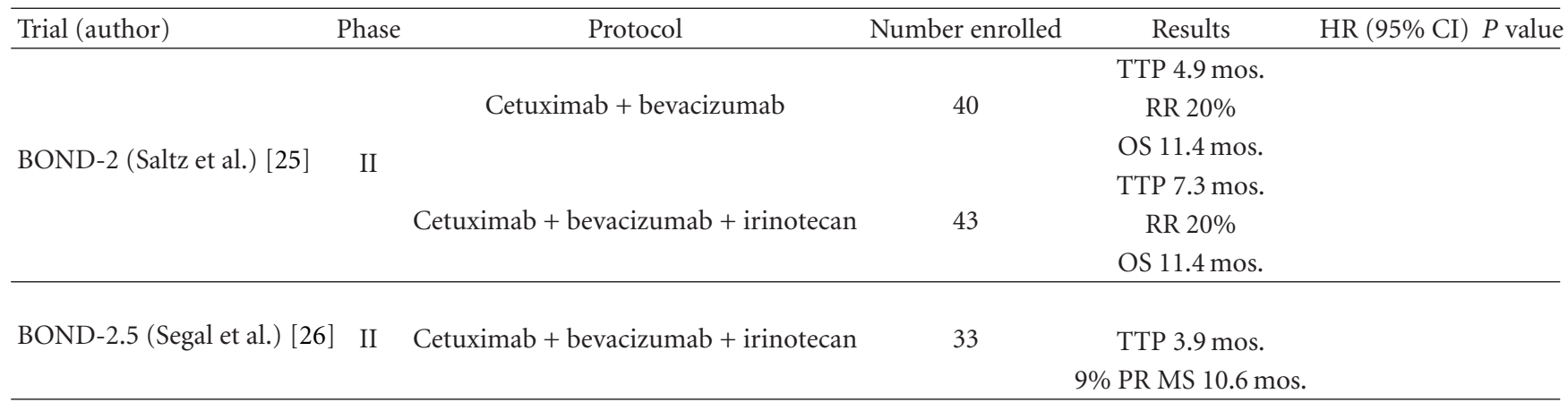

TTP: time to progression, RR: response rate, OS: overall survival, PR: partial response, and MS: median survival.

bevacizumab, an antivascular endothelial growth factor. Bevacizumab had been shown to improve RR, PFS, and OS in combination with infusional 5FU (alone or in combination with either irinotecan or oxaliplatin) as part of first- or second-line therapy of mCRC $[23,24]$.

\section{Anti-EGFR mAbs with Bevacizumab in Combination with Chemotherapy for Chemotherapy-Resistant mCRC Not Selected by KRAS (Table 5)}

In 2007, Saltz et al. evaluated the combination of cetuximab and bevacizumab in a phase II trial, BOND-2. Patients were naïve to both mAbs and were randomized to receive cetuximab plus bevacizumab with or without irinotecan (CBI or $\mathrm{CB}$, resp.). This study concluded that the mAb doublet with or without irinotecan was feasible with toxicities similar to those expected for each agent [25]. Response rate,, time to progression (TTP), and OS favored the triple drug regimen, although these results were not confirmed in a subsequent study, BOND-2.5 [26]. This study evaluated CBI in patients with $\mathrm{mCRC}$ who had previously progressed on a chemotherapy plus bevacizumab regimen. The RR and TTP seen with $\mathrm{CBI}$ did not appear to be as encouraging as the $37 \% \mathrm{RR}$ and 7.3 months TTP seen in the BOND-2 trial [26]. KRAS status was not evaluated in either BOND-2 or BOND-2.5.

\section{Chemotherapy Plus Bevacizumab with or without anti-EGFR $m A b s$ in wtKRAS Treatment Naïve mCRC (Table 6)}

Trials assessing anti-EGFR mAbs in combination with bevacizumab and chemotherapy have consistently demonstrated that double $\mathrm{mAb}$ regimens are inferior to bevacizumab and chemotherapy [27]. Tol et al. compared the combination of capecitabine, oxaliplatin, and bevacizumab with or without weekly cetuximab. They concluded that the addition of cetuximab to this trial resulted in a statistically significant decrease in PFS and inferior quality of life [27].

The PACCE trial in 2009 sought to combine drugs known to be effective in mCRC while avoiding overlapping toxicities. This trial evaluated panitumumab added to bevacizumab and chemotherapy as first-line treatment for mCRC [28]. The trial had four treatment arms: FOLFIRI plus bevacizumab plus or minus panitumumab and FOLFOX plus bevacizumab plus or minus panitumumab. Panitumumab was discontinued after a planned interim analysis of 812 FOLFOX patients demonstrated worse PFS and OS in the panitumumab arm [28]. The negative outcome of this study raised the possibility of negative interactions between EGFR inhibitors and bevacizumab when combined with chemotherapy. Notably, subjects with mutKRAS disease had a trend towards poorer survival rates when treated with panitumumab [28]. A report from a phase III study investigating capecitabine, oxaliplatin, and bevacizumab with or without cetuximab in first-line mCRC (CAIRO2) also showed inferior PFS in the investigational arm (10.7 versus 9.8 mos.; $P=0.019$ ) [30].

Saltz and colleagues compared FOLF (leucovorin $400 \mathrm{mg} / \mathrm{m}^{2}$ plus 5 -FU $400 \mathrm{mg} / \mathrm{m}^{2}$ bolus followed by 2.4 gram 48 -hour infusion every 48 hours) in combination with bevacizumab and cetuximab to FOLFOX plus bevacizumab for first-line treatment of mCRC. The authors hoped that replacing oxaliplatin with two targeted therapies would achieve superior 12-month PFS with a better tolerated regimen. The trial demonstrated that the combination of FOLF + bevacizumab + cetuximab is inferior to FOLFOX plus bevacizumab in terms of PFS at 12 months $(44.6 \%$ versus $32.3 \% ; P=0.03)$. Overall survival also was not statistically different ( 21.3 mos. versus 19.5 mos., $P=0.13$ ) although the trial was not powered for OS. Furthermore, patient satisfaction was lower in the investigational arm possibly because cetuximab specific side effects are more troubling to patients than oxaliplatin specific side effects [29]. This trial adds to evidence from the PACCE and Tol trials that combining anti-EGFR mAbs to bevacizumab based chemotherapy is an inappropriate treatment for mCRC.

\section{Recently Published Trials of Anti-EGFR mAbs Combined with Other Targeted Therapies (Table 7)}

The addition of brivanib alaninate, a TKI targeting vascular endothelial and fibroblast growth factor receptors, to cetuximab has shown encouraging activity in an early phase 


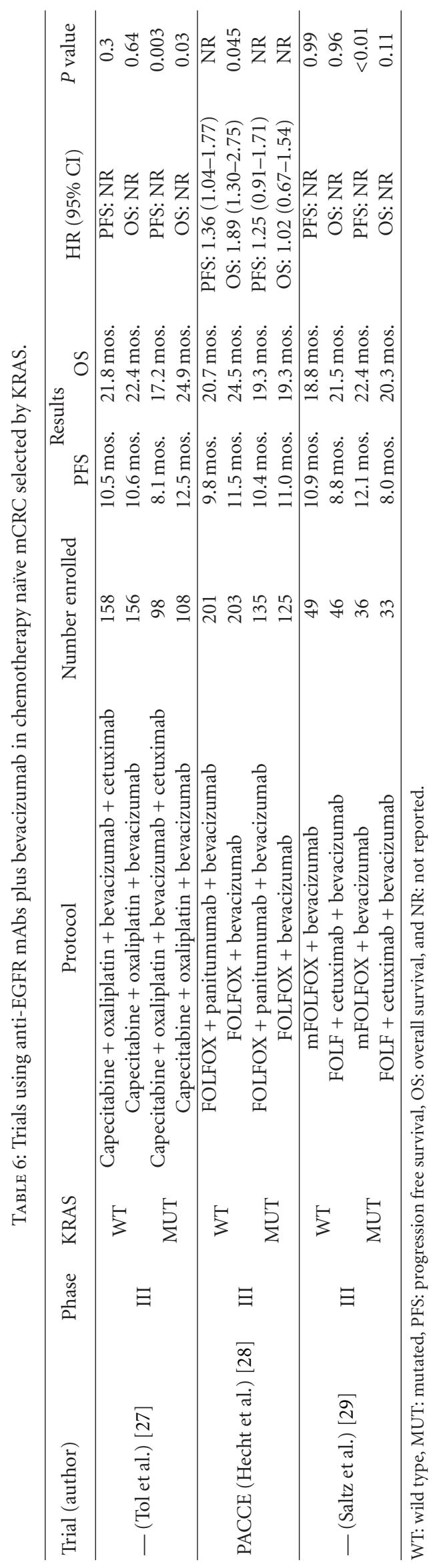


TABLE 7: Trials using anti-EGFR MAbs with other targeted therapies in chemotherapy-resistant mCRC in KRAS WT patients.

\begin{tabular}{|c|c|c|c|c|c|c|c|}
\hline \multirow{2}{*}{ Trial (author) } & \multirow{2}{*}{ Phase } & \multirow{2}{*}{ Protocol } & \multirow{2}{*}{$\begin{array}{l}\text { Number } \\
\text { enrolled }\end{array}$} & \multicolumn{2}{|c|}{ Results } & \multirow{2}{*}{$\operatorname{HR}(95 \% \mathrm{CI})$} & \multirow{2}{*}{$P$ value } \\
\hline & & & & PFS & OS & & \\
\hline \multirow{2}{*}{$\begin{array}{l}\text { AGITG CO.20 } \\
\text { (Siu et al.) [31] }\end{array}$} & \multirow{2}{*}{ III } & Cetuximab + brivanib alaninate & 376 & 5.0 mos. & 8.8 mos. & PFS: $0.72(0.62-0.84)$ & $<0.0001$ \\
\hline & & cetuximab & 374 & 3.4 mos. & 8.1 mos. & OS: $0.88(0.74-1.03)$ & 0.12 \\
\hline \multirow{3}{*}{$\frac{\text { - (Watkins et al. })}{[32]}$} & \multirow{3}{*}{ II/III } & $\begin{array}{c}\text { Dalotuzumab } 10 \mathrm{mg} / \mathrm{kg} 1 \text { week }+ \\
\text { cetuximab }+ \text { irinotecan } \\
\text { versus }\end{array}$ & Total enrolled & & 10.8 mos. & \multirow{3}{*}{$\begin{array}{l}\text { PFS: NR } \\
\text { OS: NR }\end{array}$} & \\
\hline & & $\begin{array}{c}\text { dalotuzumab } 7.5 \mathrm{mg} / \mathrm{kg} \mathrm{q} 2 \text { weeks } \\
\text { versus }\end{array}$ & $345 \mathrm{WT}$ & 5.4 mos. & 11.6 mos. & & \\
\hline & & placebo + cetuximab + irinotecan & & 5.6 mos. & 14.0 mos. & & \\
\hline
\end{tabular}

WT: wild type, PFS: progression free survival, OS: overall survival, NR: not reported.

TABLE 8: Clinical trials of cetuximab in stage III colon cancer.

\begin{tabular}{|c|c|c|c|c|c|c|}
\hline Trial (first author) & Phase & Protocol & Number enrolled & Results & $\operatorname{HR}(95 \% \mathrm{CI})$ & $P$ value \\
\hline \multirow{2}{*}{$\begin{array}{l}\text { NCCTG NO147 } \\
\text { (Goldberg et al.) [33] }\end{array}$} & \multirow{2}{*}{ III } & FOLFOX 6 + cetuximab & 318 & $\begin{array}{c}3 \text {-year DFS } 62.3 \% \\
\text { OS } 79.1 \%\end{array}$ & \multirow{2}{*}{$\begin{array}{c}\text { DFS: } 1.48(1.08-2.03) \\
\text { OS: } 1.67(1.00-2.80)\end{array}$} & \multirow{2}{*}{$\begin{array}{l}0.02 \\
0.07\end{array}$} \\
\hline & & FOLFOX 6 & 340 & $\begin{array}{c}\text { 3-year DFS } 70.3 \% \\
\text { OS } 86.1 \% \\
\end{array}$ & & \\
\hline \multirow{2}{*}{$\begin{array}{l}\text { NCCTG NO17 } \\
\text { (Huang et al.) [34] }\end{array}$} & \multirow{2}{*}{ III } & FOLFIRI + cetuximab & 45 & $\begin{array}{c}\text { 3-year DFS } 80 \% \\
\text { OS } 90 \%\end{array}$ & \multirow{2}{*}{$\begin{array}{l}\text { DFS: } 0.6(0.3-1.1) \\
\text { OS: } 0.4(0.1-1.0)\end{array}$} & \multirow{2}{*}{$\begin{array}{l}0.09 \\
0.04\end{array}$} \\
\hline & & FOLFIRI & 111 & $\begin{array}{c}\text { 3-year DFS } 65 \% \\
\text { OS } 83 \%\end{array}$ & & \\
\hline
\end{tabular}

DFS: disease-free survival, OS: overall survival.

clinical trial. However, despite positive effects on PFS and objective response rates, the combination of these two drugs did not significantly improve OS [31]. The addition of dalotuzumab, an anti-insulin-like growth factor receptor (IGFR) $\mathrm{mAb}$ to cetuximab and irinotecan worsened PFS and OS in pts with chemorefractory wtKRAS mCRC in a phase II/III study conducted by Watkins et al. [32].

\section{Cetuximab in the Adjuvant Setting (Table 8)}

Typical of drug development in oncology, once a drug demonstrates clinical benefit in metastatic disease, its efficacy is often assessed as adjuvant therapy for earlier stage, operable tumors. Intergroup study N0147 evaluated the addition of cetuximab to adjuvant FOLFOX-6 in patients who had undergone complete resection of stage III colon cancer. Worsened disease-free survival (DFS) and a trend toward inferior OS was observed in patients with mutKRAS treated with cetuximab [33]. In 2010, the same intergroup study reported updated data on 156 subjects treated on arms with FOLFIRI alone or with cetuximab. FOLFIRI resulted in a 3-year DFS lower than that expected for FOLFOX. However, trends for improved 3-year DFS (80\% versus 65\%; $\mathrm{HR}=0.6(95 \% \mathrm{CI}, 0.3$ to 1.1$) ; P=0.09)$ and 3 -year OS (90\% versus $83 \%$; $\mathrm{HR}=0.4(95 \% \mathrm{CI}, 0.1$ to 1.0$) ; P=0.04$ ) with the addition of cetuximab to FOLFIRI were observed in patients with resected stage III CRC regardless of KRAS status [34]. While only 27 subjects had wtKRAS tumors treated with adjuvant FOLFIRI plus cetuximab, the resulting 3 year DFS of $88 \%$ is provocative. These outcomes from
N0147 raise two important hypotheses: (1) the possibility that chemotherapy choice may be an important factor in the further development of anti-EGFR mAbs as adjuvant therapy and (2) KRAS mutational status may be an important factor in personalizing adjuvant treatment for resected stage III and possibly high risk-stage II CRC.

PETACC-8 a randomized, multicenter, European phase III trial is comparing the efficacy of cetuximab plus FOLFOX4 with that of FOLFOX-4 alone in patients with stage III colon cancer. The primary end point of this study is DFS time analyzed after a minimum followup of 3 years per patient [35].

\section{Monoclonal Antibodies as Neoadjuvant Therapy for Potentially Resectable Stage IVA mCRC}

The role of neoadjuvant chemotherapy for unresectable colorectal liver metastasis has been established as a method of downsizing tumors for the purpose of curative resection. Folprecht et al. conducted an open-label study randomizing patients to receive neoadjuvant cetuximab plus either FOLFOX6 or FOLFIRI (CELIM trial). The primary endpoint was RR. There was no difference in the two groups in terms of response rates (difference 11\%, 95\% CI -8-30; odds ratio (OR) $1.62,0.74-3.59 ; P=0.23$ ). Not surprisingly a retrospective analysis based on KRAS status showed that the response in the wtKRAS tumors was $70 \%$ versus $41 \%$ in the mutKRAS tumors (OR 3.42, 95\% CI 1.35-8.66; $P=0.008$ ). Furthermore, tumors that were both wtKRAS and wtBRAF 
had a $72 \% \mathrm{RR}$ as compared to the tumors that harbored a mutation in either gene $(72 \%$ versus $40 \%, P=0.003)$. The matured survival data recently reported by this group showed no difference in OS between the wtKRAS and mutKRAS groups (36.1 versus 27.4 months) however [36].

\section{Monoclonal Antibodies and Rectal Cancer}

The German Rectal Cancer Study Group reported that preoperative chemoradiation improves local control and sphincter preservation and is associated with reduced toxicity but does not improve survival compared with postoperative therapy [37]. Retrospective analyses have demonstrated lower pathologic complete response (pCR) rates and shorter DFS in patients with rectal cancer expressing EGFR who were treated with neoadjuvant radiation therapy. This suggested that radiosensitivity might be increased by targeting the EGFR [38]. Studies are investigating the role of radiation sensitizing agents and the combination of radiotherapy with targeted agents in an attempt to improve local control and DFS [37]. Phase I/II studies of combinations of cetuximab and chemoradiotherapy have demonstrated that cetuximab can be given safely, but the pCR rates have been low [38]. To date, these studies have failed to demonstrate correlation between KRAS status and efficacy of mAbs.

\section{Emerging Strategies to Identify and Overcome Anti-EGFR Resistance}

While only KRAS mutations are currently validated as predictive markers to treatment with mAbs, many wtKRAS patients still do not benefit from these drugs. Research is now concentrated on other EGFR downstream effectors, proteins that may be potential predictors of response to mAbs. BRAF proteins are downstream from KRAS and studies have shown that BRAF mutations also play a role in resistance to mAbs. The V600E is the most common point mutation that involves the BRAF gene and is present in approximately $10 \%$ of CRCs [5]. Studies looking at the response to mAbs in wtBRAF tumors have shown that tumors that are both wtKRAS and wtBRAF have better response rates, suggesting BRAF as a therapeutic target. This was demonstrated in the DUX study in which wtKRAS tumors had a $41 \%$ RR, but when wtKRAS/wtBRAF tumors were analyzed the RR was 52\% [39]. A retrospective analysis of $\mathrm{mCRC}$ patients who received therapy with EGFR mAbs showed that 11 out of $113(10 \%)$ tumors had a BRAF V600E mutation, and none of them responded to EGFR monotherapy [40].

Two studies retrospectively evaluated simultaneous mutations in KRAS and BRAF and response to mAbs. The Laurent-Puig group collected samples from 173 patients with mCRC. One hundred and sixteen of these tumors were wtKRAS, 100 of which were also wtBRAF. They found that BRAF mutations were weakly associated with lack of response $(P=0.063)$ but strongly associated with shorter PFS $(P<0.001)$ and shorter OS $(P<0.001)[41]$. Similarly, Ruzzo et al. found that among 66 wtKRAS tumors, nine or $14 \%$ were mutBRAF. Wild-type BRAF tumors had improved
RR (33\%; $P=0.04)$, and although there was a trend towards prolonged PFS (5.1 versus 3.3 mos.; $P=0.076$ ), this was not statistically significant [42].

Bokemeyer et al. analyzed the pooled data of 845 subjects with wtKRAS tumors from the Crystal and the Opus trials. BRAF mutations were present in 70 of the 800 evaluable tumors. They found that the prognosis was poorer in the mutBRAF tumors as compared to the wtBRAF tumors. The small number of mutBRAF tumors in these studies reflects the frequency of this mutation [43]. Although data to this point does not merit using BRAF mutational status to guide anti-EGFR mAbs selection, further investigation is warranted.

One of the main pathways activated by EGFR is the P13 K/PTEN/AKT signaling cascade. Mutations in the PIK3CA gene, which encodes for the p110 catalytic subunit of the PI3 K, occur in about $15 \%$ of tumors [5]. These mutations can be found together with KRAS and BRAF mutations, and this makes it difficult to define the relative contribution of this mutation to anti-EGFR mAbs resistance. Apart from KRAS and BRAF, two other groups of molecules related to the EGFR pathway have also emerged as potential biomarker candidates: EGFR ligands and fragment crystallizablegamma receptors $(\mathrm{Fc} \gamma \mathrm{R})$ polymorphisms. EGFR is activated by a variety of ligands such as amphiregulin (AREG), epiregulin (EREG), and transforming growth factor- $\alpha$. It has been shown that higher levels of AREG and EREG have a positive predictive value in determining response to cetuximab, and when treated with cetuximab these tumors have better PFS [40].

Although attempts at developing drugs targeting KRAS have largely been unsuccessful, several BRAF inhibitors have been discovered. Sorafenib is an oral multikinase inhibitor that targets both wtBRAF and oncogenic BRAF V600E and has in vitro activity in CRC cell lines with this mutation. The NEXIRI phase II trial combining sorafenib with irinotecan showed that this combination in patients with chemotherapy-resistant mutKRAS tumors has encouraging activity. Median PFS and OS were 3.5 months and 7.7 months, respectively [45]. PLX4032 (RG7204) is an oral inhibitor of the mutBRAF kinase with pronounced activity in mutBRAF melanoma patients. A phase I study of PLX4032 included a cohort of patients with mutBRAF mCRC. Clinical activity in these patients was modest (PFS 3.7 months) but does support mutant BRAF as a therapeutic target in colorectal cancers [46].

The issue of cross-resistance between panitumumab and cetuximab was evaluated in a trial of twenty patients with wtKRAS mCRC treated with cetuximab and irinotecan followed by panitumumab monotherapy after progression. No patients responded. The PFS was 1.7 months and the OS was 5.2 months. At this time, the use of panitumumab following cetuximab failure cannot be recommended [47].

\section{The Monoclonal Antibody Rash (Table 9)}

The most common side effects of anti-EGFR mAbs are dermatologic including an acne-like rash, xerosis (dry skin), and fissures of the skin. Although some degree of acneiform 
TABLE 9: The relationship between the development of rash and clinical outcome when using anti-EGFR mAbs in mCRC.

\begin{tabular}{|c|c|c|c|c|c|c|c|}
\hline \multirow{2}{*}{ Trial (first author) } & \multirow{2}{*}{ KRAS } & \multirow{2}{*}{ Protocol } & \multirow{2}{*}{ Grade of rash } & \multicolumn{2}{|c|}{ Results } & \multirow{2}{*}{$\operatorname{HR}(95 \% \mathrm{CI})$} & \multirow{2}{*}{$P$ value } \\
\hline & & & & PFS & OS & & \\
\hline \multirow{8}{*}{ — (O’Callaghan et al.) [44] } & \multirow{2}{*}{ WT } & BSC versus & \multirow{2}{*}{$0 / 1$} & 1.9 mos. & 5 mos. & PFS: $0.57(0.38-0.86)$ & 0.008 \\
\hline & & cetuximab & & 2.2 mos. & 8 mos. & OS: $0.85(0.56-1.31)$ & 0.46 \\
\hline & \multirow{2}{*}{ WT } & BSC versus & \multirow{2}{*}{$2+$} & 1.9 mos. & 5 mos. & PFS: $0.32(0.21-0.49)$ & $<0.001$ \\
\hline & & cetuximab & & 5.1 mos. & 9.8 mos. & OS: $0.52(0.34-0.8)$ & 0.003 \\
\hline & \multirow{2}{*}{ MUT } & BSC versus & \multirow{2}{*}{$0 / 1$} & 1.9 mos. & 5 mos. & PFS: 0.97 (0.65-1.46) & 0.89 \\
\hline & & cetuximab & & 1.8 mos. & 4.0 mos. & OS: $1.47(0.95-2.27)$ & 0.08 \\
\hline & \multirow{2}{*}{ MUT } & BSC versus & \multirow{2}{*}{$2+$} & 1.9 mos. & 5 mos. & PFS: $0.82(0.52-1.3)$ & 0.89 \\
\hline & & cetuximab & & 1.8 mos. & 6.6 mos. & OS: $0.82(0.47-1.41)$ & 0.46 \\
\hline
\end{tabular}

WT: wild type, MUT: mutated, PFS: progression-free survival, and OS: overall survival.

rash occurs in most patients, severe eruptions resulting in significant pain or infectious sequelae are less common [48]. Available retrospective evidence suggests that the appearance and severity of a skin rash is positively correlated with objective tumor response to mAb therapy and with OS in $\mathrm{mCRC}$ [49]. In patients receiving cetuximab or panitumumab monotherapy for mCRC, longer survival times were observed in patients with rash of any grade as compared with patients experiencing no rash [48]. Retrospectively observed rash-to-survival correlations suggest that individualized dose titration based on the appearance and severity of skin rash may allow for optimization of therapy and has led to the initiation of "dose-to-rash" trials [49]. In the EVEREST trial, patients with no or slight skin reactions after 22 days of standard cetuximab therapy were randomized to continue receiving a standard dose or to begin a dose escalation every two weeks until a grade 2 skin toxicity developed or a ceiling dose of $500 \mathrm{mg} / \mathrm{m}^{2}$ was achieved. As compared with the standard-dose arm, the dose-escalation arm demonstrated an improved RR (risk ratio: $30 \%$ versus 13\%). This finding supports the relationship between tumor RR, mAb dose, and skin rash [50]. The NCIC Clinical Trials CO.17 trial demonstrated a strong correlation between benefit from cetuximab therapy and both rash and KRAS status. Rash was graded weekly by NCI CTC 2.0 criteria. More severe rash was observed in the subjects with wtKRAS tumors than in the mutKRAS patients (57\% versus $44.4 \% ; P=0.08$ ). In addition, rash severity was positively correlated with PFS and OS in the wtKRAS patients [44].

STEPP was a phase II open-label trial of skin toxicity evaluation in mCRC patients receiving panitumumab plus FOLFIRI or irinotecan only in the chemoresistant setting. This randomized study examined differences between preemptive and reactive skin treatments for the rash associated with panitumumab therapy. Patients randomized to the preemptive treatment arm received daily skin treatment and oral doxycycline from 24 hours before their first dose of panitumumab through week 6 . Patients in the reactive treatment arm received treatment only after development of skin toxicity. As compared with the reactive treatment, preemptive treatment reduced the incidence of grade 2 or greater skin toxicities by more than $50 \%$ without additional side effects [51].

\section{Electrolyte Imbalances}

Grade 3/4 hypomagnesemia has been consistently reported across clinical trials of cetuximab and panitumumab [49]. Some data point to a direct relationship between the duration of cetuximab exposure and hypomagnesemia. The mechanisms responsible for hypomagnesemia in association with anti-EGFR mAbs have not been well defined [52]. Increased EGFR expression in the ascending loop of Henle, where 70\% of filtered magnesium is reabsorbed, may result in damage to the renal tubule, and interfere with magnesium transport, causing a magnesium wasting syndrome [52, 53]. Symptoms of hypomagnesemia can be cardiovascular, neuromuscular, or behavioral. Hypocalcemia has been reported in association with hypomagnesemia and can contribute to neuromuscular symptoms. This hypomagnesemic hypocalcemia can only be corrected by replacing magnesium levels. The pathophysiology of hypocalcemia in this setting is related to hypomagnesemia-induced PTH resistance [54].

Hypokalemia has similarly been associated with antiEGFR therapy, although to a lesser extent than hypomagnesemia. Patients with grade I hypomagnesemia are generally asymptomatic and do not require replacement therapy. In patients with grade 2 hypomagnesemia, oral supplementation is generally ineffective and poorly tolerated due to diarrhea. Weekly intravenous treatment with magnesium sulfate $4 \mathrm{~g}$ has been shown to be effective for patients with magnesium levels of 0.9 to $1.0 \mathrm{mg} / \mathrm{dL}(0.37-0.41 \mathrm{mmol} / \mathrm{L})$. For patients with grade 2 hypomagnesemia who are asymptomatic and do not have cardiac risk factors, weekly monitoring without magnesium supplementation may be considered. Grades 3-4 hypomagnesemia are associated with symptoms of fatigue, cramps and somnolence which can mistakenly be attributed to cytotoxic chemotherapy and therefore go unreported. Replacement therapy is particularly important for these patients to prevent cardiac arrhythmias and sudden cardiac death [54].

\section{Conclusion}

Although anti-EGFR targeted therapy has become an important component of the treatment of mCRC, only a subset of patients benefit from these treatments. It is becoming 


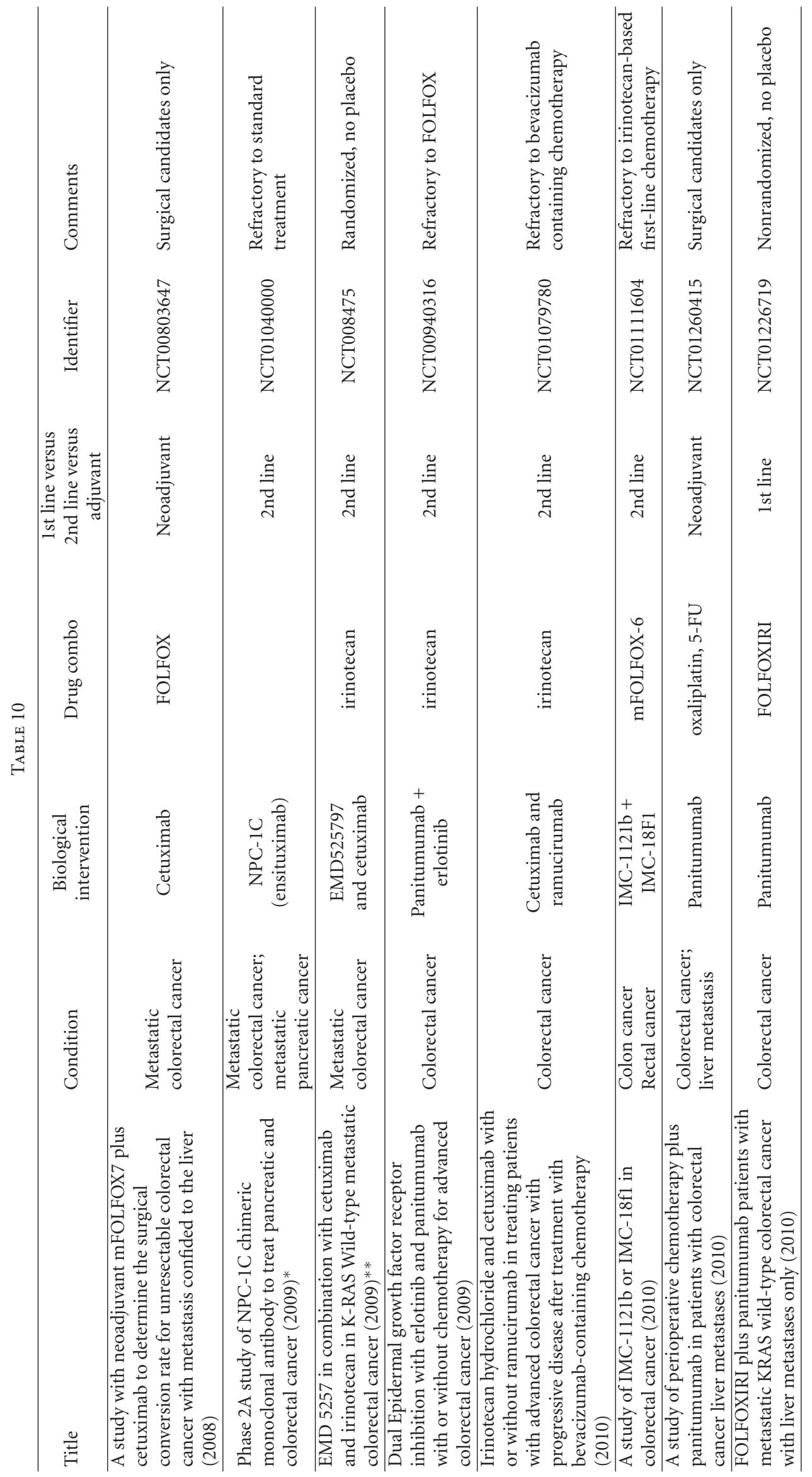




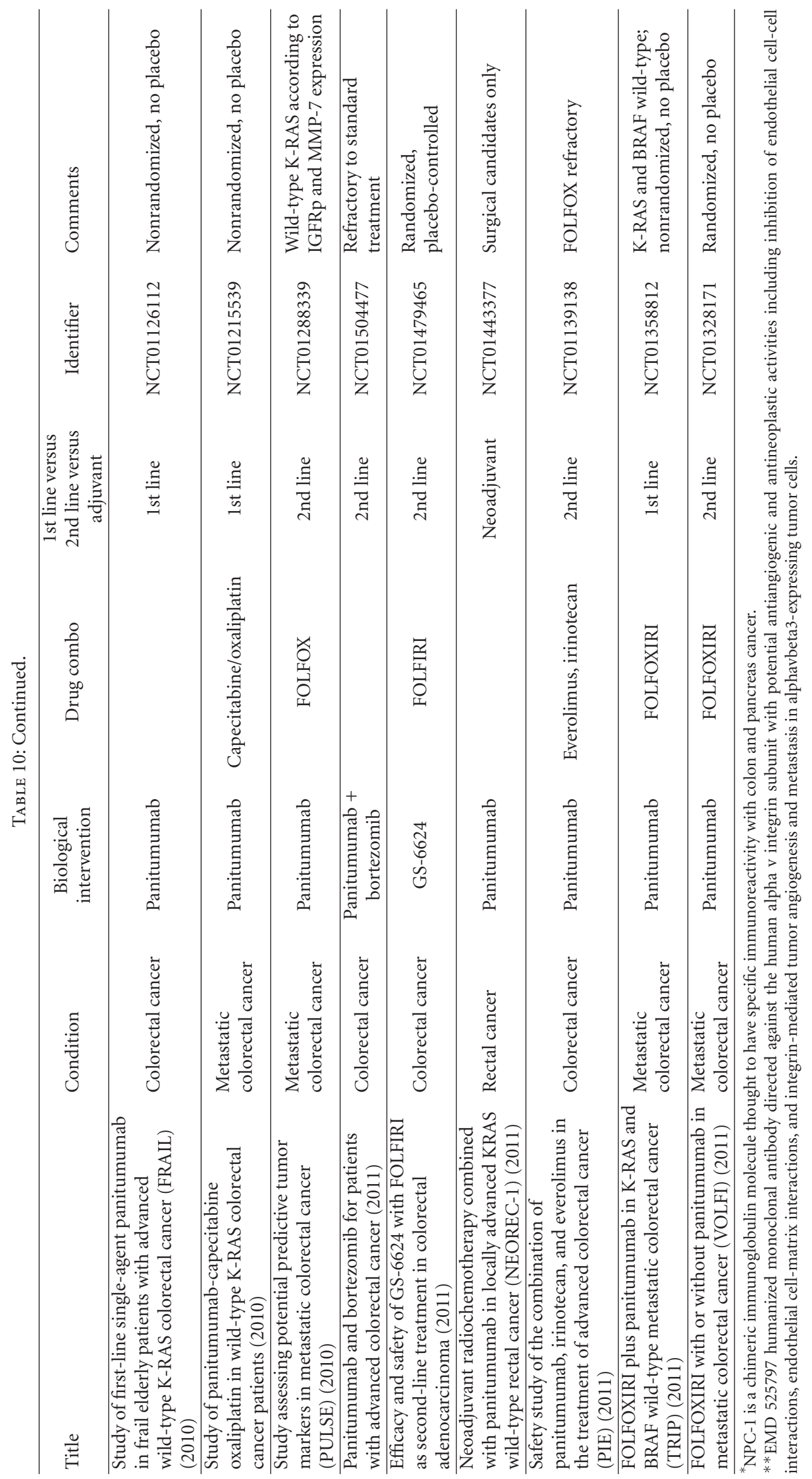


evident that KRAS mutational status is only a small aspect of why some patients respond to these treatments while others do not. There are many trials underway investigating the role of anti-EGFR antibodies either as single agents or in combination with other targeted therapies (Table 10). As summarized in this paper, clinical and translational investigators have been productive in trying to identify either optimal strategies or expanded roles for EGFR-targeted therapy as treatment for colorectal cancer. Unfortunately, despite these efforts, data to date suggests that anti-EGFR $\mathrm{mAb}$ use should be restricted to patients with wtKRAS mCRC resistant to fluorinated pyrimidines, oxaliplatin, and irinotecan (or for patients intolerant to irinotecan) either as monotherapy or in combination with irinotecan.

\section{References}

[1] N. Howlader, A. M. Noone, M. Krapcho et al., "SEER Cancer Statistics Review, 1975-2008," National Cancer Institute, Bethesda, Md, USA, 2011, http://seer.cancer.gov/csr/ 1975_2009_pops09/index.html.

[2] American Cancer Society, "Colorectal Facts \& Figures 20112013".

[3] C. R. Garrett and C. Eng, "Cetuximab in the treatment of patients with colorectal cancer," Expert Opinion on Biological Therapy, vol. 11, no. 7, pp. 937-949, 2011.

[4] K. Ng and A. X. Zhu, "Targeting the epidermal growth factor receptor in metastatic colorectal cancer," Critical Reviews in Oncology/Hematology, vol. 65, no. 1, pp. 8-20, 2008.

[5] L. Vecchionea, B. Jacobsa, N. Normannoc, F. Ciardiellod, and S. Tejpar, "EGFR-targeted therapy," Experimental Cell Research, vol. 317, no. 19, pp. 2765-2771, 2011.

[6] J. Mendelson, "Targeting the epidermal growth factor receptor for cancer therapy," Journal of Clinical Oncology, vol. 20, no. 18, supplement, pp. 1S-13S, 2002.

[7] L. B. Saltz, N. J. Meropol, P. J. Loehrer, M. N. Needle, J. Kopit, and R. J. Mayer, "Phase II trial of cetuximab in patients with refractory colorectal cancer that expresses the epidermal growth factor receptor," Journal of Clinical Oncology, vol. 25, pp. 5225-5232, 2004.

[8] D. J. Jonker, C. J. O'Callaghan, C. S. Karapetis et al., "Cetuximab for the treatment of colorectal cancer," The New England Journal of Medicine, vol. 357, pp. 2040-2048, 2007.

[9] M. Peeters, E. Van Cutsem, S. Siena et al., "A phase III, multicenter, randomized controlled trial of panitumumab plus best supportive care (BSC) vs BSC alone in patients with metastatic colorectal cancer," in Proceedings of the 97th Annual Meeting of the American Association for Cancer Research, vol. 47, April 2006, abstract no. CP-1.

[10] D. Cunningham, Y. Humblet, S. Siena et al., "Cetuximab monotherapy and cetuximab plus irinotecan in irinotecan- refractory metastatic colorectal cancer," The New England Journal of Medicine, vol. 351, no. 4, pp. 337-345, 2004.

[11] A. F. Sobrero, J. Maurel, L. Fehrenbacher et al., "EPIC: phase III trial of cetuximab plus irinotecan after fluoropyrimidine and oxaliplatin failure in patients with metastatic colorectal cancer," Journal of Clinical Oncology, vol. 26, no. 14, pp. 23112319, 2008.

[12] C. Lockhart and J. D. Berlin, "The epidermal growth factor receptor as a target for colorectal cancer therapy," Seminars in Oncology, vol. 32, no. 1, pp. 52-60, 2005.
[13] B. Markman, F. Javier Ramos, J. Capdevila, and J. Tabernero, "EGFR and KRAS in colorectal cancer," Advances in Clinical Chemistry, vol. 51, pp. 71-119, 2010.

[14] R. G. Amado, M. Wolf, M. Peeters et al., "Wild-type KRAS is required for panitumumab efficacy in patients with metastatic colorectal cancer," Journal of Clinical Oncology, vol. 26, no. 10, pp. 1626-1634, 2008.

[15] P. Bohanes, M. J. LaBonte, T. Winder, and H. J. Lenz, "Predictive molecular classifiers in colorectal cancer," Seminars in Oncology, vol. 38, no. 4, pp. 576-587, 2011.

[16] A. Lièvre, J.-B. Bachet, D. Le Corre et al., "KRAS mutation status is predictive of response to cetuximab therapy in colorectal cancer," Cancer Research, vol. 66, no. 8, pp. 3992-3995, 2006.

[17] F. A. Monzon, S. Ogino, M. E. H. Hammond, K. C. Halling, K. J. Bloom, and M. N. Nikiforova, "The role of KRAS mutation testing in the management of patients with metastatic colorectal cancer," Archives of Pathology and Laboratory Medicine, vol. 133, no. 10, pp. 1600-1606, 2009.

[18] M. T. Seymour, S. R. Brown, S. Richman et al., "Addition of panitumumab to irinotecan: results of PICCOLO, a randomized controlled trial in advanced colorectal cancer," Journal of Clinical Oncology, vol. 29, supplement, abstract no. 3523, 2011.

[19] A. F. Sobrero, M. Peeters, T. J. Price et al., "Final results from study 181: randomized phase III study of FOLFIRI with or without panitumumab for the treatment of second-line metastatic colonorectal cancer," Journal of Clinical Oncology, vol. 30, supplement 4, abstract no. 387, 2012.

[20] E. Van Cutsem, C. H. Köhne, E. Hitre et al., "Cetuximab and chemotherapy as initial treatment for metastatic colorectal cancer," The New England Journal of Medicine, vol. 360, no. 14, pp. 1408-1417, 2009.

[21] C. Bokemeyer, I. Bondarenko, A. Makhson et al., "Fluorouracil, leucovorin, and oxaliplatin with and without cetuximab in the first-line treatment of metastatic colorectal cancer," Journal of Clinical Oncology, vol. 27, no. 5, pp. 663-671, 2009.

[22] J. Y. Douillard, S. Siena, J. Cassidy et al., "Randomized, Phase III trial of panitumumab with infusional fluorouracil, leucovorin, and oxaliplatin (FOLFOX4) Versus FOLFOX4 alone as first-line treatment in patients with previously untreated metastatic colorectal cancer: the PRIME study," Journal of Clinical Oncology, vol. 28, no. 31, pp. 4697-4705, 2010.

[23] B. J. Giantonio, P. J. Catalano, N. J. Meropol et al., "Bevacizumab in combination with oxaliplatin, fluorouracil, and leucovorin (FOLFOX4) for previously treated metastatic colorectal cancer: results from the Eastern Cooperative Oncology Group Study E3200," Journal of Clinical Oncology, vol. 25, no. 12, pp. 1539-1544, 2007.

[24] H. Hurwitz, L. Fehrenbacher, W. Novotny et al., "Bevacizumab plus irinotecan, fluorouracil, and leucovorin for metastatic colorectal cancer," The New England Journal of Medicine, vol. 350, no. 23, pp. 2335-2342, 2004.

[25] L. B. Saltz, H. J. Lenz, H. L. Kindler et al., "Randomized phase II trial of cetuximab, bevacizumab, and irinotecan compared with cetuximab and bevacizumab alone in irinotecanrefractory colorectal cancer: the BOND-2 study," Journal of Clinical Oncology, vol. 25, no. 29, pp. 4557-4561, 2007.

[26] N. H. Segal, D. Reidy-Lagunes, M. Capanu et al., "Phase II study of bevacizumab in combination with cetuximab plus irinotecan in irinotecan-refractory colorectal cancer patients who have progressed on a bevacizumab-containing regimen: the BOND 2.5 Study," Journal of Clinical Oncology, vol. 27, no. 15s, supplement, abstract no. 4087, 2009. 
[27] J. Tol, M. Koopman, A. Cats et al., "Chemotherapy, bevacizumab, and cetuximab in metastatic colorectal cancer," The New England Journal of Medicine, vol. 360, pp. 563-572, 2009.

[28] J. R. Hecht, E. Mitchell, T. Chidiac et al., "A randomized phase IIIB trial of chemotherapy, bevacizumab, and panitumumab compared with chemotherapy and bevacizumab alone for metastatic colorectal cancer," Journal of Clinical Oncology, vol. 27, no. 5, pp. 672-680, 2009.

[29] L. Saltz, S. Badarinath, S. Dakhil et al., "Phase III trial of cetuximab, bevacizumab, and 5-fluorouracil/leucovorin vs. FOLFOX-bevacizumab in colorectal cancer," Clinical Colorectal Cancer, vol. 11, no. 2, pp. 101-111, 2012.

[30] C. J. Punt, J. Tol, C. J. Rodenburg et al., "Randomized phase III study of capecitabine, oxaliplatin, and bevacizumab with or without cetuximab in advanced colorectal cancer (ACC), the CAIRO2 study of the Dutch Colorectal Cancer Group (DCCG)," Journal of Clinical Oncology, vol. 26, supplement, abstract no. LBA4011, 2008.

[31] L. Siu et al., "Phase III randomized trial of cetuximab plus either brivanib alanin or placebo in patients with metastatic chemotherapy refractory K-RAS wild-type colorectal carcinoma: the NCIC Clinical Trials Group and AGITG CO. 20 Trial," Journal of Clinical Oncology, vol. 30, supplement 4, abstract no. 386, 2012.

[32] D. J. Watkins, J. Tabernero, H. Schmoll et al., "A randomized phase II/III study of the anti-IGF-1R antibody MK-0646 (dalotuzumab) in combination with cetuximab and irinotecan in the treatment of chemorefractory metastatic colorectal cancer with wild-type KRAS status2011," Journal of Clinical Oncology, vol. 29, supplement, abstract no. 3501.

[33] M. Goldberg, D. J. Sargent, S. N. Thibodeau et al., "Adjuvant mFOLFOX6 plus or minus cetuximab in patients with KRAS mutant resected stage III colon cancer: NCCTG Intergroup Phase III trial N0147," Journal of Clinical Oncology, vol. 28, no. 15s, supplement, abstract no. 3508, 2010.

[34] J. Huang, D. J. Sargent, M. R. Mahoney et al., "Adjuvant FOLFIRI with or without cetuximab in patients with resected stage III colon cancer: NCCTG Intergroup Phase III trial N0147," Journal of Clinical Oncology, vol. 29, supplement 4, abstract no. 363, 2011.

[35] J. Taieb, P. L. Puig, and L. Bedenne, "Cetuximab plus FOLFOX-4 for fully resected stage III colon carcinoma: scientific background and the ongoing PETACC-8 trial," Expert Review of Anticancer Therapy, vol. 8, no. 2, pp. 183-189, 2008.

[36] G. Folprecht, T. Gruenberger, W. O. Bechstein et al., "Tumour response and secondary resectability of colorectal liver metastases following neoadjuvant chemotherapy with cetuximab: the CELIM randomised phase 2 trial," The Lancet Oncology, vol. 11, no. 1, pp. 38-47, 2010.

[37] J. P. Machiels, C. Sempoux, P. Scalliet et al., "Phase I/II study of preoperative cetuximab, capecitabine, and external beam radiotherapy in patients with rectal cancer," Annals of Oncology, vol. 18, no. 4, pp. 738-744, 2007.

[38] K. M. Sullivan and P. S. Kozuch, "Impact of KRAS mutations on management of colorectal carcinoma: pathology Research International," vol. 2011, Article ID 219309, 11 pages, 2011.

[39] A. J. Weickhardt, T. J. Price, N. Pavlakis et al., "DUX study: a phase II study of evaluating dual targeting of the EGFR using the combination of cetuximab and erlotinib in patients with chemotherapy refractory metastatic colorectal cancer," Journal of Clinical Oncology, vol. 28, no. 15s, supplement, abstract no. 3533, 2010.

[40] A. Dasari and W. A. Messersmith, "New strategies in colorectal cancer: biomarkers of response to epidermal growth factor receptor monoclonal antibodies and potential therapeutic targets in phosphoinositide 3-kinase and mitogen-activated protein kinase pathways," Clinical Cancer Research, vol. 16, no. 15, pp. 3811-3818, 2010.

[41] P. Laurent-Puig, A. Cayre, G. Manceau et al., "Analysis of PTEN, BRAF, and EGFR status in determining benefit from cetuximab therapy in wild-type KRAS metastatic colon cancer," Journal of Clinical Oncology, vol. 27, no. 35, pp. 59245930, 2009.

[42] A. Ruzzo, C. Cremolini, F. Loupakis et al., "Association of BRAF mutations and EGFR Intron-1 L/L genotype with resistance to cetuximab plus irinotecan treatment in KRAS wild-type metastatic colon cancer patients," Journal of Clinical Oncology, vol. 27, no. 15s, supplement, abstract no. 4058, 2009.

[43] C. Bokemeyer, E. V. Cutsem, P. Rougier et al., "Addition of cetuximab to chemotherapy as first-line treatment for KRAS wild-type metastatic colorectal cancer: pooled analysis of the CRYSTAL and OPUS randomized clinical trials," European Journal of Cancer, vol. 48, no. 10, pp. 1466-1475, 2012.

[44] C. J. O'Callaghan, D. Tu, C. S. Karapetis et al., “The relationship between the development of rash and clinical and quality of life outcomes by KRAS mutation status in patients with colorectal cancer treated with cetuximab in NCIC CTG CO.17," Journal of Clinical Oncology, vol. 29, supplement 4, abstract no. 481, 2011.

[45] M. Ychou, O. Bouche, S. Thézenas et al., "Final results of a multicenter phase II trial assessing sorafenib in combination with irinotecan as second-or later-line treatment in metastatic colorectal cancer patients with KRAS-mutated tumors: NEXIRI2011," Journal of Clinical Oncology, vol. 29, supplement, abstract no. e14002, 2011.

[46] S. Kopetz, J. Desai, E. Chan et al., "PLX4032 in metastatic colorectal cancer patients with mutant BRAF tumors," Journal of Clinical Oncology, vol. 28, no. 15s, supplement, abstract no. 3534, 2010.

[47] R. C. Wadlow, A. F. Hezel, T. A. Abrams et al., "Panitumumab in patients with KRAS wild-type colorectal cancer after progression on cetuximab," Oncologist, vol. 17, no. 1, 14 pages, 2012.

[48] B. M. Wolpin, J. A. Meyerhardt, H. J. Mamon, and R. J. Mayer, "Adjuvant treatment of colorectal cancer," CA Cancer Journal for Clinicians, vol. 57, no. 3, pp. 168-185, 2007.

[49] B. Melosky, R. Burkes, D. Rayson, T. Alcindor, N. Shear, and M. Lacouture, "Management of skin rash during EGFRtargeted monoclonal antibody treatment for gastrointestinal malignancies: Canadian recommendations," Current Oncology, vol. 16, no. 1, pp. 16-26, 2009.

[50] E. Van Cutsem, Y. Humblet, H. Gelderblom et al., "Cetuximab dose-escalation study in patients with metastatic colorectal cancer with no or slight skin reactions on cetuximab standard dose treatment (EVEREST): pharmacokinetic and efficacy data of randomized study," in Proceedings of the Gastrointestinal Cancer Symposium, 2007, abstract no. 237.

[51] E. P. Mitchell, M. Lacouture, H. Shearer et al., "Final STEPP results of prophylactic versus reactive skin toxicity treatment for panitumumab-related skin toxicity in patients with metastatic colorectal cancer," Journal of Clinical Oncology, vol. 27, no. 15s, supplement, abstract no. CRA4027, 2009.

[52] M. Fakih, "Management of anti-EGFR-targeting monoclonal antibody-induced hypomagnesemia," Oncology, vol. 22, no. 1, pp. 74-76, 2008. 
[53] D. Schrag, K. Y. Chung, C. Flombaum, and L. Saltz, "Cetuximab therapy and symptomatic hypomagnesemia," Journal of the National Cancer Institute, vol. 97, no. 16, pp. 1221-1224, 2005.

[54] M. Fakih and M. Vincent, "Adverse events associated with anti-EGFR therapies for the treatment of metastatic colorectal cancer," Current Oncology, vol. 17, supplement 1, pp. S18-S30, 2010. 


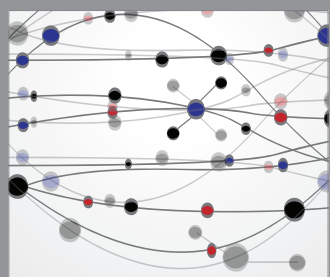

The Scientific World Journal
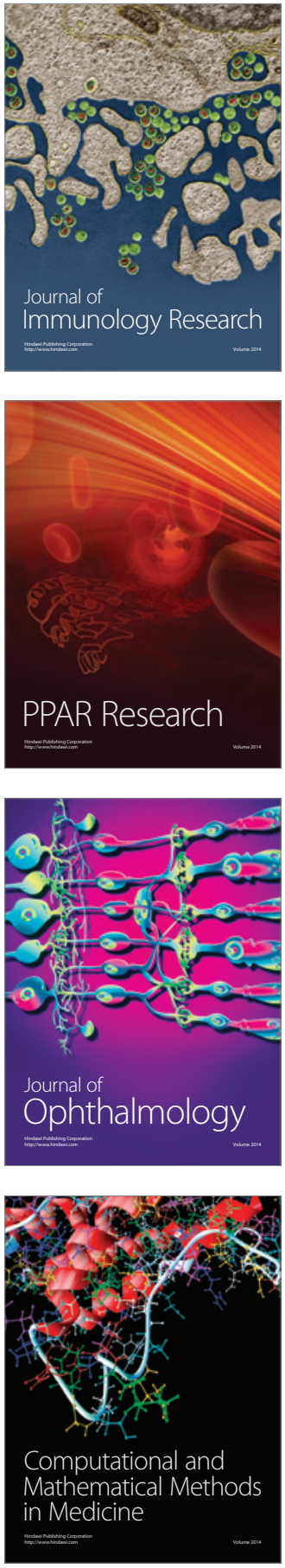

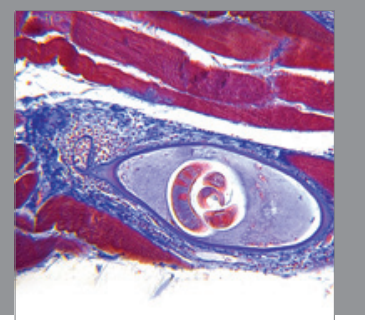

Gastroenterology

Research and Practice
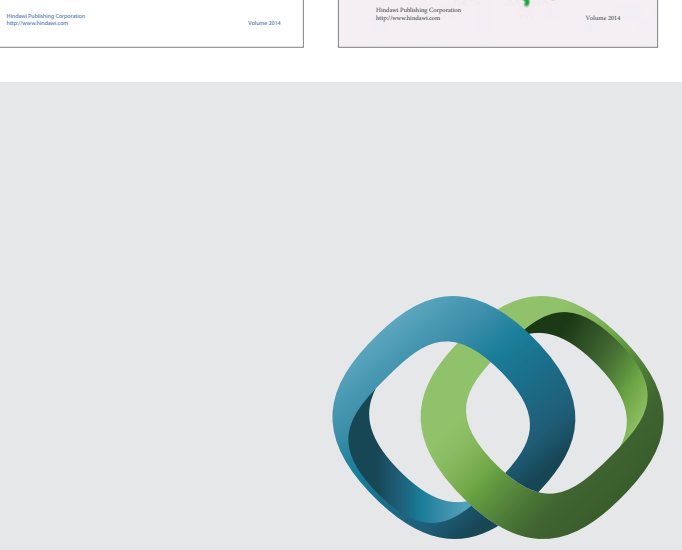

\section{Hindawi}

Submit your manuscripts at

http://www.hindawi.com
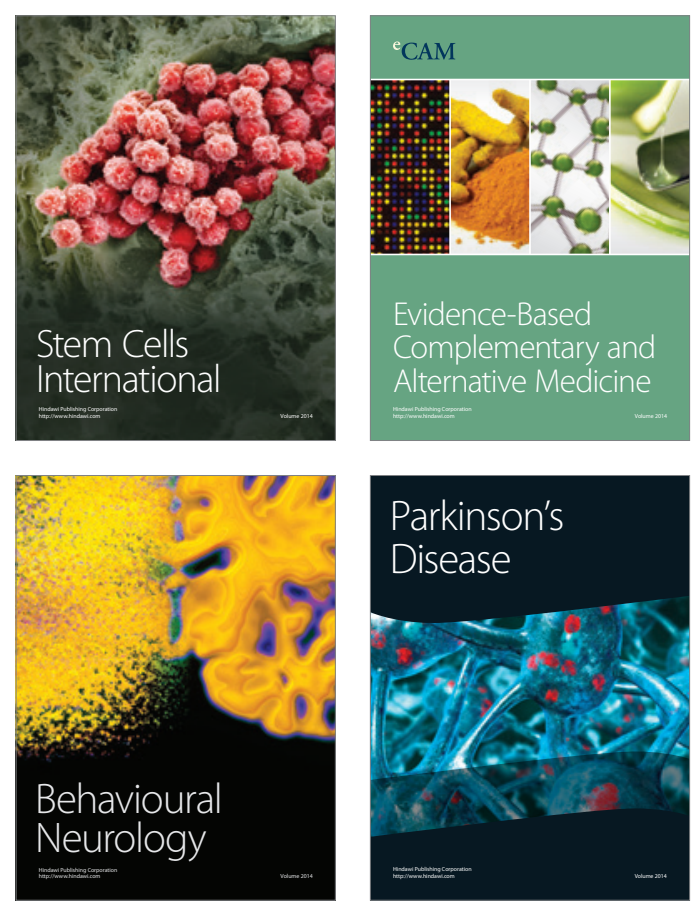

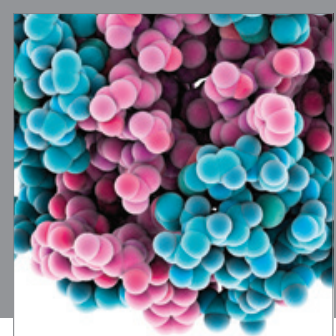

Journal of
Diabetes Research

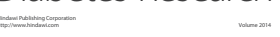

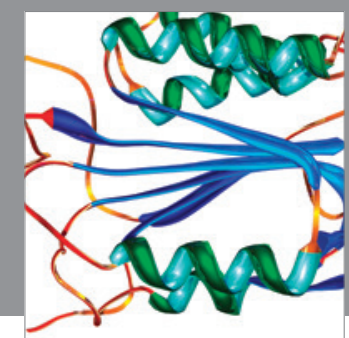

Disease Markers
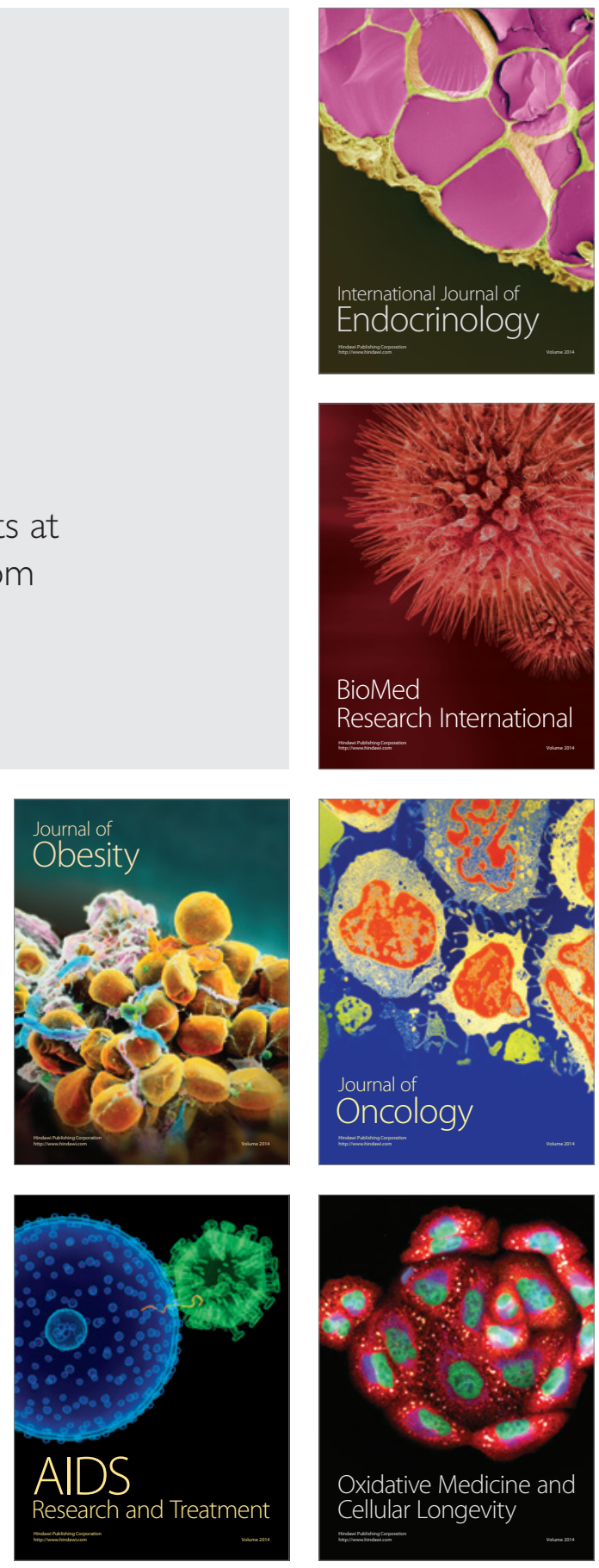